\title{
Assessment of genetic structure in Mexican charolais herds using microsatellite markers
}

\author{
Ana María Sifuentes-Rincón* \\ Laboratorio de Biotecnología Animal I \\ Centro de Biotecnología Genómica \\ Instituto Politécnico Nacional \\ Boulevard del Maestro, esq. Elías Piña \\ Col. Narciso Mendoza, Cd. Reynosa \\ C.P. 88710, Tam. México \\ Tel: 5289992436 27, Ext. 87723 \\ Fax: 528999251656 \\ E-mail: asifuentes@ipn.mx
}

\section{Herlinda Puentes-Montiel}

Laboratorio de Biotecnología Animal I

Centro de Biotecnología Genómica Instituto Politécnico Nacional Boulevard del Maestro, esq. Elías Piña, Col. Narciso Mendoza

Cd. Reynosa, Tam. México. C.P. 88710

Tel: 528999243627

Fax: 528999251656

E-mail: pmherlin@hotmail.com

\author{
Gaspar Manuel Parra-Bracamonte \\ Laboratorio de Biotecnología Animal I \\ Centro de Biotecnología Genómica \\ Instituto Politécnico Nacional \\ Boulevard del Maestro, esq. Elías Piña, Col. Narciso Mendoza \\ Cd. Reynosa, Tam. México. C.P. 88710 \\ Tel: 528999243627 \\ Fax: 528999251656 \\ E-mail: gparra@ipn.mx
}

Financial support: We acknowledge financial support from CGPI-IPN Research Grant.

Keywords: charolais, genetic diversity, microsatellite.

\author{
Abbreviations: HW: Hardy-Weinberg \\ PCR: polymerase chain reaction \\ PIC: polymorphic information content
}

Knowledge of livestock genetic diversity is an essential step to respond to commercial demands and reach production objectives in different environments and production systems. The evaluation of animal genetic diversity is achieved by using molecular markers. Microsatellites are the most used markers for studies of this type. Eleven microsatellites were used to evaluate the genetic variation from three populations of Charolais cattle located in northeast Mexico. The studied populations exhibited a high allelic variability with a mean heterozygosity of 0.5 . A moderate genetic differentiation between the Charolais populations $\left(\mathrm{F}_{\mathrm{ST}}=\right.$ 0.079; $\mathbf{P}<0.001)$ was observed. This suggests subdivisions in Charolais breed established in Mexico, due to genetic material origin, reproductive and selective management and local isolation.
Breeding and conservation programs can be determined by characterizing the genetic variation of livestock (Notter, 1999). Genetic improvement may reduce significantly the genetic variation within populations (Vasconcellos et al. 2003). To date, there are an increasing number of specialized breeds spread mainly for the development of reproduction systems (i.e. artificial insemination, embryo transfer, etc.) conducting to a reduction on their population effective size and complicating sustained genetic improvement (Mapletoft and Hasler, 2005). The Holstein breed is a clear example of widespread germplasm for milk production improvement. An evident consequence is a large Holstein population maintained only for a few sires. In such cases genetic diversity evaluation, is primordial in order to avoid the deleterious consequences (Notter, 1999; Williams, 2005).

*Corresponding author 
Microsatellites are an important tool in population genetic studies. They are highly polymorphic, presents simple mendelian inheritance and codominance (segregation of homozygotes and heterozygotes (Boichard et al. 1998)).

Versatility for adaptation to a wide range of management conditions has made the Charolais a highly used beef cattle breed in the world. In was imported to Mexico in the 1930 's. The best animals have been selected for performance and phenotypic characteristics. Because the breed is so adaptable to varying conditions, it has been used in almost all states of the country as purebred or crossbred cattle.

Genetic diversity of the Charolais developed in Mexico can be a consequence of several factors. Some of these factors are the differences in management and in selective decisions, and the use of imported genetic material. The study of genetic variation in different populations will eventually allow the understanding of the effect of germplasm introduction, gene flow and/or selection movements. The objective of the present study was to evaluate the genetic molecular variation at three founder Charolais populations from different environments in Mexico.

\section{MATERIALS AND METHODS}

Whole blood was collected from one hundred-ninety four non-related purebred Charolais cattle in three ranches. Two ranches are located in Nuevo Leon, and one in Veracruz, Mexico. The selection criterion at each ranch is breed pattern, conformation, and weight gain. These ranches are national distributors of the Charolais genetics. Each ranch was identified as a subpopulation, according with the origin of genetic material used for improvement. Ranch 1, imported genetic material only from France; ranch 2, utilizes its own and local genetic material; and ranch 3 imports genetic material from France, UK and Ireland.

Isolation of DNA was done as is reported by Peelman et al. (1998). Genetic diversity was determined by using eleven microsatellites loci; Table 1 describes loci sequences and polymerase chain reaction (PCR) conditions. Amplification profiles consisted of one cycle at $95^{\circ} \mathrm{C}$ during $10 \mathrm{~min}$; five three-step cycles of $45 \mathrm{sec}$ at $95^{\circ} \mathrm{C}, 45 \mathrm{sec}$ at $62^{\circ} \mathrm{C}$ (temperature was reduced $2^{\circ} \mathrm{C}$ each cycle), $45 \mathrm{sec}$ at $72^{\circ} \mathrm{C}$; 25 cycles with the following steps each one of $45 \mathrm{sec}$ at $95^{\circ} \mathrm{C}, 55^{\circ} \mathrm{C} 45 \mathrm{sec}$ (this temperature was changed for each loci according with Table 1), $45 \mathrm{sec}$ at $72^{\circ} \mathrm{C}$; and finally one cycle of $10 \mathrm{~min}$ at $72^{\circ} \mathrm{C}$. PCR products were denaturized at $95^{\circ} \mathrm{C}$ for $5 \mathrm{~min}$, and then electrophoresed on a $6.5 \%$ denaturing polyacrylamide-bisacrilamide gel during $2 \mathrm{hrs}$ on a LI-COR sequencer (LI-COR, Inc. Lincoln, Nebraska USA) model 42001G.

Allele size for each locus was obtained using SAGA GT ${ }^{\mathrm{TM}}$ software (LI-COR, Inc. Lincoln, Nebraska USA). Numbers of alleles (k) observed (HO) and expected (HE) heterozygosity, allelic frequencies, and the polymorphic information content (PIC) for each locus and in combinations of loci were estimated using CERVUS 2.0 software (Marshall et al. 1998). Diversity analysis was performed using the same software. Population structure was established by an Analysis of Molecular Variance (AMOVA), using Arlequin (Excoffier et al. 2005). Effective number of alleles was estimated according to $\mathrm{Li}$ et al. (2004).

\section{RESULTS AND DISCUSSION}

All loci showed high polymorphism in all ranches (Table 2). Twelve alleles per loci was the average. Allele number per locus ranged from 7 alleles in ETH10 locus, to 18 alleles in TGLA53. The allele diversity was similar among the ranches. Ranch 2 showed a unique allelic series for marker ETH10 (from 203 bp to 217 bp). ETH10 locus also showed the most different allele number between ranches. Ranches had unique alleles. Ranch 2 had seven at five loci, meanwhile the ranches 1 and 3, presented six and five, respectively.

Expected heterozygocity was higher than the observed for all loci in the three ranches. The overall mean heterozygocity was 0.500 . Observed heterozygosity ranged from 0.388 to 0.780 , from 0.235 to 0.816 , and from 0.230 to 0.732 for ranches 1,2 , and 3 , respectively. Heterozygosity is a reliable estimator of genetic diversity (MacHugh et al. 1998). MacHugh et al. (1998) reported a value of heterozygosity for Charolais of 0.525 . Maudet et al. (2002) found a heterozygosity of 0.640 in Charolais in France. These heterozygocity values are similar than those estimated in the present study, suggesting the genetic diversity of Charolais in Mexico is comparable to that reported in Europe.

An overall departure from Hardy-Weinberg expectation (HW) was ascertained in the present study for all markers with the exception of BMS1886 ( $<0.01$; Table 3$)$. Deviation of genetic equilibrium, might suggest a subdivision in the whole population due to a Wahlund effect (Hartl and Clark, 1997).

Based on the analysis of molecular variance (Table 4), there was a moderate differentiation $\left(\mathrm{F}_{\mathrm{ST}}=0.079 ; \mathrm{P}<0.001\right)$ among ranches (Hartl and Clark, 1997). Variation within and between the ranches was of 92.03 and $7.97 \%$, respectively. Jordana et al. 2003, reported an analysis in eight Southwest European beef cattle breeds, their results showed a genetic differentiation between breeds of $6.8 \%$, authors pointing out that those values are similar to those founded in humans, dogs, sheep, goats, rabbits and pigs. Similarly, another study in European beef cattle, reported, values of differentiation between breeds of $7.0 \%$; this degree of differentiation was related to a relatively low genetic flow and high reproductive isolation (Cañón et al. 2001). Moioli et al. (2004), reported between isolated Italian breeds an estimate of $\mathrm{F}_{\mathrm{ST}}=0.06$, even lower than the 
one found for our studied subpopulations. Considering the similarity of our results with those previously found between breeds even species, they could be an evidence of subdivisions within the Mexican Charolais breed, suggesting a direct consequence of genetic material origin and reproductive or selective management practices.

Fernández et al. (2006) suggested that subdivision of an animal population may have negative effects conducting to a reduced population size and, therefore, it could generate higher inbreeding levels and a depression at fitness-related traits. One way to avoid that side-effect is to allow certain degree of gene flow between subpopulations. However, Notter (1999), indicated that the maintenance of allelic diversity is achieved in subdivided rather than panmictic populations so long these are large enough to pass through the inbreeding effects, or managed for an occasional crossing to renew heterozygosity. Application of these concepts to evaluated populations, could avoid the inbreeding effects, a recommendation is to continue with the current reproduction practices, which are based on using imported semen.

These results show the relationships at the evaluated populations, not the complete history of the breed in Mexico. Further studies must be done in order to confirm the subdivisions within Charolais breed in Mexico and its implications in the design of crossbreeding strategies focused to exploit the productive advantages of each subdivided breed-population.

\section{ACKNOWLEDGMENTS}

Authors thank to Dr. Eduardo Casas for his helpful comments and manuscript revision, we also thank to Gilberto Romero Cloud, Ricardo Maldonado González and Martín del Ángel Robles, for the technical assistance and support obtaining the samples.

\section{REFERENCES}

BOICHARD, D.; LE ROY, P.; LEVEZIEL, H. and ELSEN, J.-M. Utilisation des marqueurs moléculaires en génétique animale. INRA Productions Animales, 1998, vol. 11, no. 1, p. 67-80.

CAÑON, Javier; ALEXANDRINO, Paolo; BESSA, Isabel; CARLEOS, Carlos; CARRETERO, Yolanda; DUNNER, Susana; FERRAND, Nuno; GARCIA, David; JORDANA, Jordi; LALOE, Denis; PEREIRA, Albano; SANCHEZ, Armand and MOAZAMI-GOUDARZI, Katayoun. Genetic diversity measures of local European beef cattle breeds for conservation purposes. Genetics Selection and Evolution, May-June 2001, vol. 33 no. 3, p. 311-332.

EXCOFFIER, Laurent; LAVAL, Guillaume and SCHNEIDER, Stefan. Arlequin (version 3.0): An integrated software package for population genetics data analysis. Evolutionary Bioinformatics, 2005, vol. 1, p. 4750 .
FERNANDEZ, M.J.; TORO, M.A. and CABALLERO, A. Development of a dynamic management system of subdivided populations in conservation programmes. In: Electronic memories of $8^{\text {th }}$ World Congress on Genetics Applied to Livestock Production $\left(13^{\text {th }}-18^{\text {th }}\right.$ August, Belo Horizonte, MG, Brasil). 2006.

HARTL, Daniel and CLARK, Andrew G. Principles of population genetics. $3^{\text {th }}$ ed. Sunderland MA, Sinauer Associates Inc., 1997. 542 p. ISBN 0-87-893306-9.

JORDANA, Jordi; ALEXANDRINO, Paolo; BEJAPEREIRA, Albano; BESSA, Isabel; CAÑON, Javier; CARRETERO, Yolanda; DUNNER, Susana; LALOE, Denis; MOAZAMI-GOUDARZI, Katayoun; SANCHEZ, Albano and FERRAND, Nuno. Genetic structure of eighteen local south European beef cattle breeds by comparative $F$-statistics analysis. Journal of Animal Breeding and Genetics, April 2003, vol. 120, no. 2, p. 7387.

LI, S.-J.; YANG, S.-H.; ZHAO, S.-H.; FAN, B.; YU, M.; WANG, H.-S.; LI, M.-H.; LIU, B.; XIONG, T.-A. and LI, $\mathrm{K}$. Genetic diversity analyses of 10 indigenous Chinese pig populations based on 20 microsatellites. Journal of Animal Science, February 2004, vol. 82, no. 2, p. 368-374.

MACHUGH, D.E.; LOFTUS, R.T.; CUNNINGHAM, P. and BRADLEY, D.G. Genetic structure of seven European cattle breeds assessed using 20 microsatellite markers. Animal Genetics, October 1998, vol. 29, no. 5, p. 333-340.

MAPLETOFT, R.J. and HASLER, J.F. Assisted reproductive technologies in cattle: a review. Revue Scientifique et Technique - Office International des Epizooties, April 2005, vol. 24, no. 1, p. 393-403.

MARSHALL, T.C.; SLATE, J.; KRUUK, L.E.B. and PEMBERTON, J.M. Statistical confidence for likelihoodbased paternity inference in natural populations. Molecular Ecology, May 1998, vol. 7, no. 5, p. 639-655.

MAUDET, C.; LUIKART, G. and TABERLET, P. Genetic diversity and assignment tests among seven French cattle breeds based on microsatellite DNA analysis. Journal of Animal Science, April 2002, vol. 80, no. 4, p. 942-950.

MOIOLI, B.; NAPOLITANO, F. and CATILLO, G. Genetic diversity between Piedmontese, Maremmana and Podolica Cattle Breeds. Journal of Heredity, 2004, vol. 95, no. 3 , p. $250-256$

NOTTER, D.R. The importance of genetic diversity in livestock populations of the future. Journal of Animal Science, January 1999, vol. 77, no. 1, p. 61-69.

PEELMAN, L.J.; MORTIAUX, F.; VAN ZEVEREN, A.; DANSERCOER, A.; MOMMENS, G.; COOPMAN, F.; BOUQUET, Y.; BURNY, A.; RENAVILLE, R. and PORTETELLE, D. Evaluation of the genetic variability of 
23 bovine microsatellite markers on four Belgian cattle breeds. Animal Genetics, June 1998, vol. 29, no. 3, p. 161167.

VASCONCELLOS, Luciana Pimentel de Mello Klocker; TAMBASCO-TALHARI, Daniella; PEREIRA, Andréa Pozzi; COUTINHO, Luiz Lehmann and REGITANO, Luciana Correia de Almeida. Genetic characterization of Aberdeen Angus cattle using molecular markers. Genetics and Molecular Biology, 2003, vol. 26, no. 2, p. 133-137.

WILLIAMS, J.L. The use of marker-assisted selection in animal breeding and biotechnology. Revue Scientifique et Technique - Office International des Epizooties, April 2005, vol. 24, no. 1, p. 379-391. 


\section{APPENDIX}

\section{TABLES}

Table 1. Chromosomal location, primer sequences and PCR conditions for microsatellite loci used in this study.

\begin{tabular}{|c|c|c|c|c|}
\hline \multirow[b]{2}{*}{ Locus } & \multirow[b]{2}{*}{$\mathrm{Ch}^{1}$} & \multicolumn{2}{|c|}{ PCR Conditions } & \multirow[b]{2}{*}{ Primer Sequences } \\
\hline & & $\mathrm{MgCl}_{2}{ }^{2}$ & $\begin{array}{c}\text { Annealing } \\
\text { Temperature } \\
\left({ }^{\circ} \mathrm{C}\right)^{3}\end{array}$ & \\
\hline BM2123 & 2 & 3.0 & 55 & $\begin{array}{l}\text { 5'-GCTGCCTTCTACCAAATACCC-3' } \\
\text { 5'-CTTCCTGAGAGAAGCAACACC-3' }\end{array}$ \\
\hline ETH10 & 5 & 3.0 & 55 & $\begin{array}{l}\text { 5'-GTTCAGGACTGGCCCTGCTAACA-3' } \\
\text { 5'-CCTCCAGCCCACTTTCTCTTCTC-3' }\end{array}$ \\
\hline INRA37 & 10 & 3.0 & 50 & $\begin{array}{l}\text { 5'-GATCCTGCTTATATTTTAACCAC-3' } \\
\text { 5'-AAAATTCCATGGAGAGAGAAAC-3' }\end{array}$ \\
\hline BM1824 & 1 & 1.2 & 55 & $\begin{array}{l}\text { 5'-GAGCAAGGTGTTTTTCCAATC-3' } \\
\text { 5'-CATTCTCCAACTGCTTCCTTG-3' }\end{array}$ \\
\hline BMS1886 & 2 & 3.0 & 60 & $\begin{array}{l}\text { 5'-CAGGGACTGAAAAATAATGCC-3' } \\
\text { 5'-TTCCATGTTGATTGTTTCTTCC-3' }\end{array}$ \\
\hline TGLA53 & 16 & 2.0 & 50 & $\begin{array}{l}\text { 5'-GCTTTCAGAAATAGTTTGCATTCA-3' } \\
\text { 5'-ATCTTCACATGATATTACAGCAGA-3' }\end{array}$ \\
\hline ILSTS005 & 5 & 0.8 & 50 & $\begin{array}{c}\text { 5'-GGAAGCAATGAAATCTATAGCC-3' } \\
\text { 5'-TGTTCTGTGAGTTTGTAAGC-3' }\end{array}$ \\
\hline BMS1987 & 2 & 3.0 & 60 & $\begin{array}{c}\text { 5'-TGATGCAGAGAACGTTTTAATTT-3' } \\
\text { 5'-CTTGGGGTAGGCAGAGATTT-3' }\end{array}$ \\
\hline INRA23 & 3 & 3.0 & 50 & $\begin{array}{l}\text { 5'-GAGTAGAGCTACAAGATAAACTTC3' } \\
\text { 5'-TAACTACAGGGTGTTAGATGAACTC-3' }\end{array}$ \\
\hline HEL5 & 21 & 1.0 & 50 & $\begin{array}{l}\text { 5'-GCAGGATCACTTGTTAGGGA-3' } \\
\text { 5'-AGACGTTAGTGTACATTAAC-3' }\end{array}$ \\
\hline TGLA44 & 2 & 3.0 & 65 & $\begin{array}{l}\text { 5'-AACTGTATATTGAGAGCCTACCATG-3' } \\
\text { 5'-CACAACTTAGCGACTAAACCACCA-3' }\end{array}$ \\
\hline
\end{tabular}

${ }^{1}$ Chromosome on which the microsatellite is located.

${ }^{2} \mathrm{MgCl}_{2}$ concentration (mM).

${ }^{3}$ Anneling temperature during PCR. 
Table 2. Allele frequency of eleven microsatellite loci for three studied Charolais populations.

\begin{tabular}{|c|c|c|c|c|c|c|c|c|c|c|c|}
\hline \multirow{2}{*}{$\begin{array}{l}\text { Locus/Allele } \\
\text { size }\end{array}$} & \multicolumn{3}{|c|}{$\begin{array}{c}\text { Subpopulation/Allele } \\
\text { frequency }\end{array}$} & \multirow{2}{*}{$\begin{array}{l}\text { Locus/Allele } \\
\text { size }\end{array}$} & \multicolumn{3}{|c|}{$\begin{array}{c}\text { Subpopulation/Allele } \\
\text { frequency }\end{array}$} & \multirow{2}{*}{$\begin{array}{l}\text { Locus/Allele } \\
\text { size }\end{array}$} & \multicolumn{3}{|c|}{$\begin{array}{c}\text { Subpopulation/Allele } \\
\text { frequency }\end{array}$} \\
\hline & 1 & 2 & 3 & & 1 & 2 & 3 & & 1 & 2 & 3 \\
\hline$B M 2113$ & & & & BMS1886 & & & & INRA23 & & & \\
\hline 125 & 0.0900 & 0.0256 & & 131 & 0.0612 & & 0.0070 & 193 & & & 0.0390 \\
\hline 127 & 0.0200 & 0.0513 & 0.0380 & 133 & 0.4694 & 0.4394 & 0.4437 & 195 & & & 0.0519 \\
\hline 129 & 0.0600 & 0.0641 & 0.0063 & 135 & & 0.0152 & & 197 & 0.0204 & 0.0676 & 0.0584 \\
\hline 131 & 0.3300 & 0.2949 & 0.0190 & 137 & & 0.0303 & 0.0141 & 199 & 0.0306 & 0.0135 & 0.1948 \\
\hline 133 & 0.1200 & 0.1282 & 0.3481 & 139 & 0.0102 & & 0.0352 & 201 & 0.1122 & 0.0135 & 0.0649 \\
\hline 135 & 0.1800 & 0.1538 & 0.2215 & 143 & 0.0102 & & & 203 & 0.1224 & 0.0946 & 0.2143 \\
\hline 137 & 0.1900 & 0.1923 & 0.1646 & 145 & 0.0102 & 0.0606 & 0.0141 & 205 & 0.1224 & 0.1892 & 0.1429 \\
\hline 139 & 0.0100 & 0.0256 & 0.1519 & 147 & 0.1122 & 0.1061 & 0.0423 & 207 & 0.2755 & 0.1081 & 0.0390 \\
\hline 141 & & 0.0641 & 0.0380 & 149 & 0.1327 & 0.1970 & 0.1761 & 209 & 0.0816 & 0.1892 & 0.0584 \\
\hline \multirow[t]{3}{*}{143} & & & 0.0127 & 151 & 0.0612 & 0.0455 & 0.0423 & 211 & 0.0306 & 0.0811 & 0.0779 \\
\hline & & & & 153 & 0.0102 & 0.0303 & 0.0704 & 213 & 0.0714 & 0.0946 & 0.0325 \\
\hline & & & & 155 & 0.0510 & 0.0606 & 0.0915 & 215 & 0.0408 & 0.0405 & 0.0195 \\
\hline ETH10 & & & & 157 & 0.0714 & 0.0152 & 0.0634 & 217 & 0.0204 & 0.0811 & 0.0065 \\
\hline 203 & & 0.0113 & & & & & & 219 & 0.0612 & 0.0135 & \\
\hline 205 & & 0.0113 & & TGLA53 & & & & 221 & 0.0102 & & \\
\hline 209 & & 0.1857 & 0.2000 & 145 & & & 0.0072 & 223 & & 0.0135 & \\
\hline 211 & & 0.2138 & 0.7467 & 147 & & & 0.0217 & & & & \\
\hline 213 & 0.2128 & 0.0578 & 0.0267 & 149 & 0.0900 & & 0.0072 & & & & \\
\hline 215 & 0.6809 & 0.0227 & 0.0133 & 151 & 0.1200 & 0.1429 & 0.0725 & HEL5 & & & \\
\hline \multirow[t]{3}{*}{217} & 0.1064 & 0.0113 & 0.0133 & 153 & 0.1500 & & 0.0580 & 159 & 0.0104 & & \\
\hline & & & & 155 & 0.0900 & 0.0143 & 0.1304 & 161 & 0.0417 & 0.0556 & 0.0878 \\
\hline & & & & 157 & 0.1300 & 0.2143 & 0.2536 & 163 & 0.0521 & & \\
\hline INRA37 & & & & 159 & 0.0400 & 0.1000 & 0.1232 & 165 & 0.4792 & 0.0694 & 0.1757 \\
\hline 114 & & 0.0263 & & 161 & 0.0300 & 0.0429 & 0.0725 & 167 & 0.0208 & 0.2083 & 0.0338 \\
\hline 118 & & & 0.0065 & 163 & 0.900 & 0.0857 & 0.0725 & 169 & & 0.1528 & \\
\hline 120 & & 0.0395 & 0.0260 & 165 & 0.0700 & 0.1571 & 0.0290 & 171 & & 0.0278 & \\
\hline 122 & & & 0.0260 & 167 & 0.1000 & 0.1143 & 0.0797 & 173 & & 0.0139 & \\
\hline 124 & & & 0.1364 & 169 & 0.0400 & 0.0429 & 0.0290 & 175 & 0.0417 & & 0.0676 \\
\hline 126 & 0.2857 & 0.2237 & 0.2338 & 171 & 0.0300 & & & 177 & 0.0313 & 0.0139 & 0.0541 \\
\hline 128 & 0.3265 & 0.2500 & 0.1688 & 173 & 0.0200 & 0.0286 & & 179 & 0.3229 & 0.1806 & 0.0541 \\
\hline 130 & 0.0612 & 0.0526 & 0.1299 & 175 & & 0.0286 & 0.0435 & 181 & & 0.0972 & 0.0135 \\
\hline 132 & 0.2245 & 0.2763 & 0.1948 & 177 & & 0.0143 & & 183 & & 0.0694 & 0.2095 \\
\hline 134 & 0.0612 & 0.0921 & 0.0325 & 183 & & 0.0143 & & 185 & & 0.0139 & 0.2770 \\
\hline 138 & & & 0.0130 & & & & & 187 & & 0.0972 & 0.0270 \\
\hline 144 & 0.0102 & 0.0263 & 0.0065 & ILST005 & & & & & & & \\
\hline \multirow[t]{3}{*}{146} & 0.0306 & 0.0132 & 0.0260 & 179 & & 0.0128 & & & & & \\
\hline & & & & 181 & & 0.1282 & 0.0800 & TGLA44 & & & \\
\hline & & & & 183 & 0.1735 & 0.4359 & 0.4133 & 141 & & & 0.0133 \\
\hline BM1824 & & & & 185 & 0.4592 & 0.3718 & 0.5000 & 143 & 0.0213 & 0.0469 & 0.0600 \\
\hline 174 & & 0.0405 & & 187 & 0.3673 & & & 145 & & 0.0156 & 0.0600 \\
\hline 176 & & 0.1081 & & 189 & & 0.0256 & 0.0067 & 147 & & & 0.0133 \\
\hline 178 & 0.2300 & 0.2568 & 0.0400 & 191 & & 0.0128 & & 159 & & 0.0156 & 0.0067 \\
\hline 180 & 0.1900 & 0.3784 & 0.3000 & 193 & & 0.0128 & & 161 & 0.2128 & 0.1250 & 0.2266 \\
\hline 182 & 0.2800 & 0.0541 & 0.2200 & & & & & 163 & 0.0532 & 0.1094 & 0.1267 \\
\hline 184 & 0.0200 & 0.0135 & 0.2600 & BMS1987 & & & & 165 & 0.5213 & 0.2500 & 0.3200 \\
\hline 186 & & 0.0811 & 0.0067 & 108 & & & 0.0063 & 167 & 0.1064 & 0.2344 & 0.1400 \\
\hline 188 & 0.1600 & 0.0676 & 0.0333 & 110 & & 0.0921 & 0.2250 & 169 & 0.0638 & 0.1406 & 0.0333 \\
\hline 190 & 0.1200 & & 0.1133 & 112 & 0.5300 & 0.3947 & 0.2750 & 171 & 0.0106 & 0.0469 & \\
\hline \multirow[t]{6}{*}{192} & & & 0.0267 & 114 & 0.0300 & 0.0263 & 0.0437 & 175 & 0.0106 & 0.0156 & \\
\hline & & & & 118 & & 0.0132 & & & & & \\
\hline & & & & 120 & & 0.1053 & 0.1937 & & & & \\
\hline & & & & 122 & 0.4100 & 0.2237 & 0.2250 & & & & \\
\hline & & & & 124 & & 0.0263 & 0.0188 & & & & \\
\hline & & & & 126 & 0.0300 & 0.1184 & 0.0125 & & & & \\
\hline
\end{tabular}


Table 3. Expected and observed heterozygosity $\left(H_{E}\right.$ and $H_{O}$ respectively), effective number of alleles ( $\left.a_{e}\right)$, HW deviation significance, and PIC of eleven microsatellite markers used in Charolais cattle.

\begin{tabular}{|c|c|c|c|c|c|c|c|c|c|c|c|c|}
\hline & \multicolumn{12}{|c|}{ Locus $^{1}$} \\
\hline & & 1 & 2 & 3 & 4 & 5 & 6 & 7 & 8 & 9 & 10 & 11 \\
\hline \multicolumn{13}{|c|}{ Subpopulation 1} \\
\hline \multirow[t]{5}{*}{$n^{2}=1 / 79$} & $H_{E}$ & 0.804 & 0.485 & 0.761 & 0.800 & 0.741 & 0.911 & 0.631 & 0.555 & 0.870 & 0.665 & 0.671 \\
\hline & $H_{O}$ & 0.520 & 0.000 & 0.592 & 0.780 & 0.755 & 0.680 & 0.388 & 0.560 & 0.469 & 0.688 & 0.532 \\
\hline & $H W^{3}$ & $* * *$ & $* * *$ & $* *$ & NS & NS & NS & $* * *$ & NS & $* * *$ & ** & NS \\
\hline & $a_{e}$ & 4.901 & 1.922 & 4.046 & 4.812 & 3.757 & 10.163 & 2.660 & 2.218 & 7.224 & 2.927 & 2.976 \\
\hline & $P I C$ & 0.769 & 0.426 & 0.712 & 0.760 & 0.712 & 0.893 & 0.546 & 0.453 & 0.849 & 0.605 & 0.627 \\
\hline \multicolumn{13}{|c|}{ Subpopulation 2} \\
\hline \multirow[t]{5}{*}{$n=8 / 45$} & $H_{E}$ & 0.834 & 0.651 & 0.807 & 0.774 & 0.757 & 0.885 & 0.663 & 0.769 & 0.891 & 0.880 & 0.843 \\
\hline & $H_{O}$ & 0.410 & 0.235 & 0.605 & 0.514 & 0.636 & 0.714 & 0.436 & 0.816 & 0.649 & 0.167 & 0.344 \\
\hline & $H W$ & $* * *$ & $* * *$ & $* * *$ & ** & NS & ** & ** & NS & $* * *$ & $* * *$ & $* * *$ \\
\hline & $a_{e}$ & 5.675 & 2.792 & 4.920 & 4.224 & 3.924 & 7.851 & 2.891 & 4.150 & 8.246 & 7.557 & 5.884 \\
\hline & $P I C$ & 0.803 & 0.578 & 0.768 & 0.732 & 0.719 & 0.860 & 0.590 & 0.728 & 0.867 & 0.854 & 0.809 \\
\hline \multicolumn{13}{|c|}{ Subpopulation 3} \\
\hline \multirow[t]{5}{*}{$n=17 / 63$} & $H_{E}$ & 0.781 & 0.404 & 0.846 & 0.783 & 0.755 & 0.880 & 0.577 & 0.788 & 0.877 & 0.834 & 0.813 \\
\hline & $H_{O}$ & 0.253 & 0.093 & 0.636 & 0.6 & 0.732 & 0.493 & 0.44 & 0.663 & 0.558 & 0.257 & 0.467 \\
\hline & $H W$ & $* * *$ & $* * *$ & $* * *$ & $* * *$ & NS & $* * *$ & $* * *$ & $* * *$ & $* * *$ & $* * *$ & $* * *$ \\
\hline & $a_{e}$ & 4.467 & 1.670 & 6.253 & 4.498 & 3.992 & 7.909 & 2.344 & 4.611 & 7.616 & 5.713 & 5.197 \\
\hline & $P I C$ & 0.744 & 0.356 & 0.820 & 0.743 & 0.727 & 0.863 & 0.483 & 0.748 & 0.856 & 0.804 & 0.784 \\
\hline \multicolumn{13}{|c|}{ Population } \\
\hline \multirow[t]{5}{*}{$n=26 / 187$} & $H_{E}$ & 0.802 & 0.700 & 0.820 & 0.827 & 0.751 & 0.901 & 0.420 & 0.667 & 0.549 & 0.369 & 0.461 \\
\hline & $H_{O}$ & 0.369 & 0.096 & 0.616 & 0.636 & 0.719 & 0.608 & 0.420 & 0.667 & 0.549 & 0.369 & 0.461 \\
\hline & $H W$ & $* *$ & ** & $* *$ & ** & NS & $* *$ & $* *$ & $* *$ & ** & $* *$ & $* *$ \\
\hline & $a_{e}$ & 4.981 & 3.310 & 5.476 & 5.702 & 3.977 & 9.843 & 2.896 & 3.932 & 9.922 & 7.522 & 4.700 \\
\hline & $P I C$ & 0.773 & 0.665 & 0.793 & 0.803 & 0.751 & 0.901 & 0.657 & 0.748 & 0.891 & 0.854 & 0.762 \\
\hline
\end{tabular}


Sifuentes-Rincón, A.M. et al.

Table 4. Analysis of Molecular Variance in Charolais cattle herds in northeast Mexico.

\begin{tabular}{|l|c|c|c|c|}
\hline \multicolumn{1}{|c|}{ Source of variation } & Degrees of freedom & Sum Square & Variance components & Variation \% \\
\hline Between populations & 2 & 76.971 & 0.32372 & 7.97 \\
\hline Within populations & 335 & 1252.254 & 3.73807 & 92.03 \\
\hline Total & 337 & 1329.225 & 4.06179 & \\
\hline & F & & & \\
\hline
\end{tabular}

